\title{
Study on the influence of test solution concentration on the chemical corrosion resistance of ceramic tiles
}

\author{
Tao Tian ${ }^{1}$, Shanyu Liu*, Rong Wang ${ }^{1}$, Ying Lei ${ }^{1}$, Changlin Zheng ${ }^{1}$ \\ ${ }^{1}$ China Building Material Test \& Certification Group(Shaanxi) Co. Ltd, Xi'an, Shaanxi, China, 710049 \\ *China Building Material Test \& Certification Group(Shaanxi) Co. Ltd, Xi'an, Shaanxi, China, 710049
}

\begin{abstract}
Chemical resistance is one of the important evaluation factors of ceramic tiles. This article describes the method for determining the chemical resistance of ceramic tiles, and discusses the influence of acid-base solution concentration on the test results during the experiment, and proposes corresponding improvement measures.
\end{abstract}

\section{Preface}

Ceramic products have been widely used in the general decoration, wall and floor of buildings at home and abroad because of their strong anti-corrosion, powerful pollution resistance, long service life and low price. The current product standard for ceramic tiles in CHINA is GB/T 4100-2015, and the corresponding testing method standard is GB/T3810 series. The quality evaluation indicators for evaluating ceramic tile products include appearance size, deformation, Bibulous rate, Fracture strength, wear resistance, thermal shock resistance, chemical corrosion resistance, etc. among which chemical corrosion resistance is one of the important quality inspection indexes for ceramic brick products. In this paper, the influence of the concentration of acid-base test solution on the experimental results of the chemical corrosion resistance of ceramic tiles is discussed, and the corresponding improvement measures are put forward.

\section{Test of Ceramic tiles chemical corrosion resistance}

2.1 The principle and equipment of the experiment of chemical corrosion resistance of ceramic tiles

In the experiment, the sample is directly affected by the test solution, and the degree of chemical corrosion will be observed and determined after a certain period of time under certain conditions. The equipment used in the experiment mainly includes:

Balance (precision is $0.05 \mathrm{~g}$ ); Oven (operating temperature $\left.110^{\circ} \mathrm{C} \pm 5^{\circ} \mathrm{C}\right)$, Biochemical incubator $\left(20^{\circ} \mathrm{C}\right.$ $\pm 2^{\circ} \mathrm{C}$ ); Cylinder (made of borosilicate glass); Suede etc.

\subsection{Reagents and methods for chemical corrosion resistance test of ceramic tiles}

\section{2. 1 Test reagent for chemical corrosion resistance of ceramic tiles}

Ceramic tile testing standard GB/T 3810. 13-2016 stipulates that the experimental reagents mainly include household chemicals, swimming pool salts and acid-base solutions of different concentrations, etc. In the experiment, we used hydrochloric acid solution with volume fraction of $0.01,0.02,0.03,0.04,0.05,0.16$, $0.17,0.18,0.19,0.20$, and potassium hydroxide solution with concentration of $20 \mathrm{~g} / \mathrm{L}, 25 \mathrm{~g} / \mathrm{L}, 30 \mathrm{~g} / \mathrm{L}$, $35 \mathrm{~g} / \mathrm{L}, 40 \mathrm{~g} / \mathrm{L}, 90 \mathrm{~g} / \mathrm{L}, 95 \mathrm{~g} / \mathrm{L}, 100 \mathrm{~g} / \mathrm{L}, 105 \mathrm{~g} / \mathrm{L}$, $110 \mathrm{~g} / \mathrm{L}$, respectively, to study the influence of different concentrations of strong acid and alkali test solution on the chemical corrosion resistance of ceramic tiles.

\section{2. 2 Experimental method of chemical corrosion resistance of ceramic tiles}

\section{2. 2. 1 Test method for chemical corrosion resistance of unglazed brick}

Three kinds of unglazed bricks produced by different manufacturers were selected and named as sample 1, sample 2 and sample 3 . After cleaning the surface of the unglazed brick sample with the size of $50 \mathrm{~mm} \times 50 \mathrm{~mm}$ and measuring weight, the samples were immersed in a container with test solution at a vertical depth of $25 \mathrm{~mm}$. The non cutting edge of the sample must be completely immersed in the solution and kept at $20{ }^{\circ} \mathrm{C}{ }^{\circ} \mathrm{C} \pm 2{ }^{\circ} \mathrm{C}$ for 12 days after covering the lid. After 12 days, the samples were rinsed with flowing water for 5 days. After rinsing, the samples were completely soaked in water and boiled for 30 minutes. Then they were removed from the water 
and gently wiped with wring dry but still wet suede, and then dried in a $110^{\circ} \mathrm{C} \pm 5^{\circ} \mathrm{C}$ drying oven.

\section{2. 2. 2 Experimental method of chemical corrosion resistance of glazed tiles}

The glazed bricks produced by three different manufacturers in the market were also selected for the experiment and named as samples 4, 5 and 6. Place the cylinder used to hold the reagent on the surface of the glazed ceramic tile samples that have been cleaned and sealed effectively, and mark it separately. The test liquid was injected at the opening with a liquid level of $20 \mathrm{~mm} \pm 1 \mathrm{~mm}$ to make the sample contact with the test liquid for 4days. The device was gently shaken once a day to ensure that the liquid level of the test liquid remained unchanged. After 2days, we replaced the solution, removed the cylinder after another 2 days and thoroughly cleaned the sealing material on the glaze with appropriate solvent.

\section{Influence of Test Solution Concentration on the Test Results of Ceramic Tiles' Chemical Resistance}

According to the test standard GB/T 3810. 13-2016, hydrochloric acid test solution and potassium hydroxide test solution with volume fraction of 0.03 and 0.18 and concentration of $30 \mathrm{~g} / \mathrm{L}$ and $100 \mathrm{~g} / \mathrm{L}$, respectively, are usually selected as the strong acid and strong base test solution for the test of chemical corrosion resistance of ceramic bricks. In this experiment, based on the standard concentration, a series of acid test solutions with the concentration error of \pm 0.01 and \pm 0.02 with the standard acid test solution and the series of alkaline test solution with the error of \pm 5 and \pm 10 with the standard alkali test solution are selected as the experimental solutions. The detection situation when the test solution concentration deviation occurs in the experiment is simulated, and the influence of different strong acid and strong alkali test solution concentrations on the chemical corrosion resistance of ceramic tiles is studied. According to the grading method of the test standard, the experimental results are shown in Table 1 Table 4.

Table 1 The influence of the volume fraction of the low-concentration hydrochloric acid test solution on the surface detection results of ceramic tiles

Hydrochloric acid test
liquid integral number

A-*: Means the chemical corrosion resistance is between $\mathrm{A}$ and $\mathrm{B}$

A- -* Means the chemical corrosion resistance is between A- and B 
Table 2 The influence of the volume fraction of high-concentration hydrochloric acid test solution on the surface detection results of ceramic tiles

\begin{tabular}{|c|c|c|c|c|c|}
\hline $\begin{array}{l}\text { Sample test } \\
\text { results }\end{array}$ & 0.16 & 0.17 & 0.18 & 0.19 & 0.20 \\
\hline Sample 1 & UHA & UHA & UHA & UHA & UHA- \\
\hline Sample 2 & UHA & UHA & UHA & UHA & UHA- \\
\hline Sample 3 & UHA & UHA & UHA & UHA- - & UHA- - \\
\hline Sample 4 & GHA & GHA & GHA & GHA & GHA - \\
\hline Sample 5 & GHA & GHA & GHA & GHA & GHA - \\
\hline Sample 6 & GHA & GHA & GHA & GHA & GHA- \\
\hline
\end{tabular}

Table 3 Influence of the concentration of low concentration potassium hydroxide test solution on the surface test results of ceramic brick samples

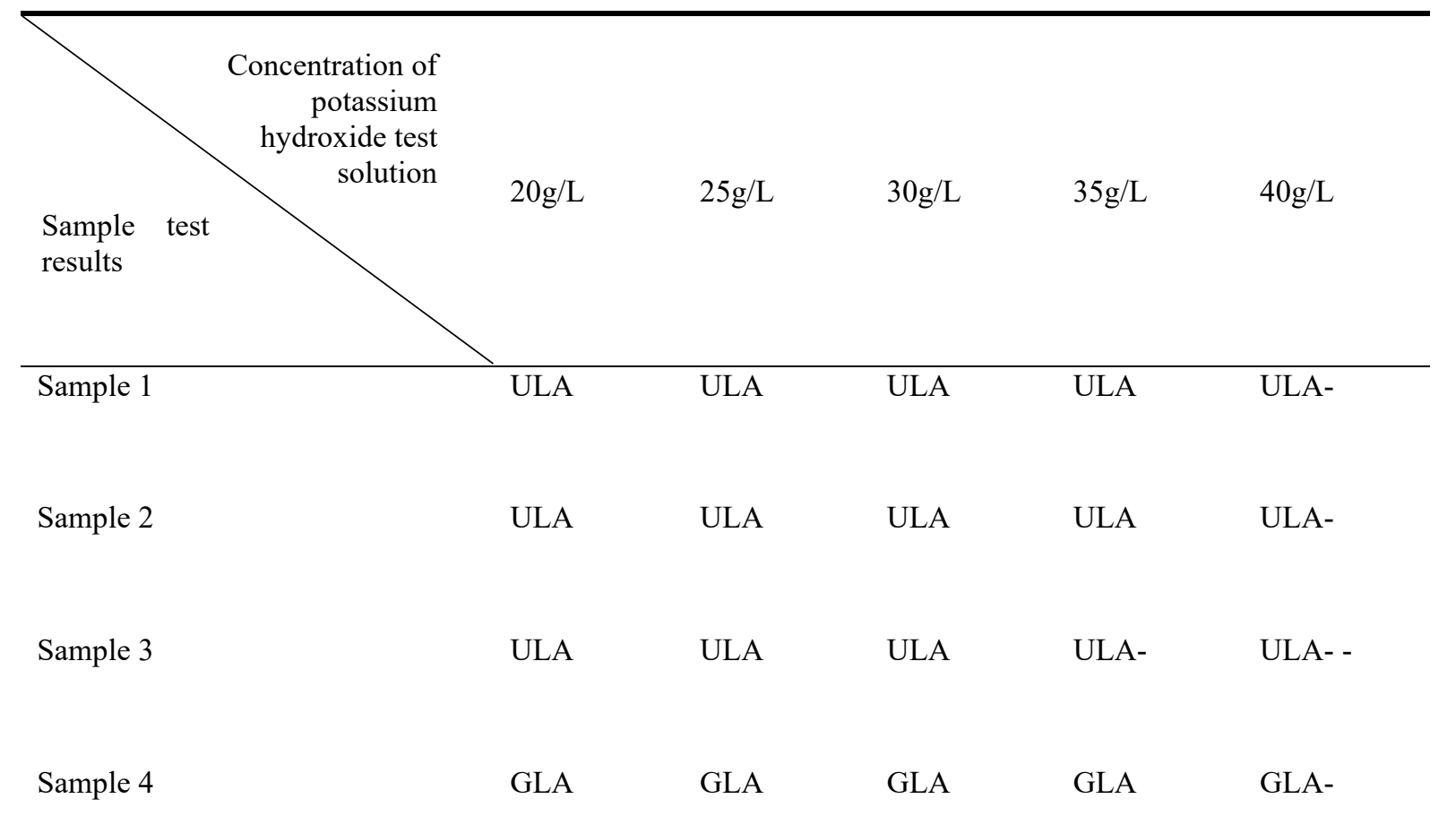


Sample 5 GLA GLA GLA GLA
Sample 6
GLA
GLA
GLA
GLA
GLA-

Table 4 The influence of the concentration of high concentration potassium hydroxide test solution on the surface test results of ceramic tiles

\begin{tabular}{|c|c|c|c|c|c|}
\hline $\begin{array}{l}\text { Sompentration of } \\
\text { potassium } \\
\text { hydroxide test } \\
\text { solution }\end{array}$ & $90 \mathrm{~g} / \mathrm{L}$ & $95 \mathrm{~g} / \mathrm{L}$ & $100 \mathrm{~g} / \mathrm{L}$ & $105 \mathrm{~g} / \mathrm{L}$ & $110 \mathrm{~g} / \mathrm{L}$ \\
\hline Sample 1 & UHA & UHA & UHA & UHA & UHA- \\
\hline Sample 2 & UHA & UHA & UHA & UHA & UHA- \\
\hline Sample 3 & UHA & UHA & UHA & UHA- - & UHA- - \\
\hline Sample 4 & GHA & GHA & GHA & GHA & GHA- \\
\hline Sample 5 & GHA & GHA & GHA & GHA & GHA- \\
\hline Sample 6 & GHA & GHA & GHA & GHA & GHA- \\
\hline
\end{tabular}

\section{Analysis and improvement of experimental results}

\section{1 Analysis of experimental results}

From the experimental results in Table 1 to table 4, we found that when using low concentration strong acid and strong base solution as the test solution, with the increase of acid-base test solution concentration, when the concentration of test solution exceeds the standard concentration, the acid-base test solution will cause slightly more serious corrosion on the sample than the standard concentration acid-base test solution, and the corrosion resistance test result grade of the corresponding sample also slightly decreases;and when the high concentration of strong acid and strong base solution is used as the test solution, with the increase of the concentration of acid-base test solution, the change trend of sample detection results is consistent with that of using low concentration strong acid and strong base solution as experimental solution. The experimental results show that the concentration of strong acid and alkali test solution in the chemical corrosion resistance test of ceramic tiles will affect the test results of samples. We should strictly control the concentration of strong acid and strong alkali test solution in the experiment according to the inspection standards of ceramic tiles.

\section{2 Improvement measures}

According to the inspection standards, the concentration of strong acid and strong base test solution in the experiment is mainly affected by the preparation steps of the solution and the operation of the inspectors. 
Therefore, we propose the following improvements:

1: When preparing the test solution, the standard solution within the validity period and the measured instrument shall be used for preparation in strict accordance with the operation instruction for preparation of test solution. After preparation, the test solution shall be stored in strict accordance with the conditions required for preservation of test solution to ensure the accuracy of test solution concentration.

2: The inspection personnel shall be trained and take up the post after passing the operation level examination. In the process of the experiment, pay attention to the control of the "volume" of the test solution to ensure the accuracy of the volume of the test solution.

\section{References}

1. GB/T 3810. 13-2016, Methods of test for ceramic tiles Part 13: Determination of chemical resistance [S].

2. GB/T 4100-2015, Ceramic tiles [S].

3. F. Han,Application of medical ceramic tiles in the decoration of infectious disease hospitals $[\mathrm{J}]$. Foshan Ceramics,30(07):8-9(2020)

4. L. Hu,Discussion on influencing Factors of determination of chemical corrosion resistance of Ceramic Brick [J]. Quality Exploration, 13(07): 47-50 (2016)

5. C. Li,Pay attention to the training of inspectors and improve the level of quality management [J]. Chemical quality, 03:39-42(2004) 